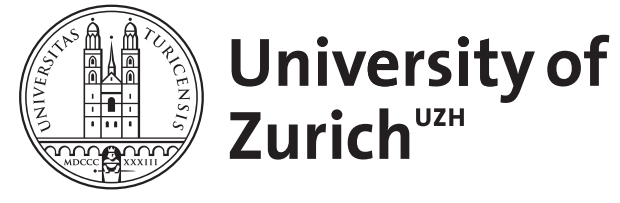
Archive

University of Zurich

University Library

Strickhofstrasse 39

CH-8057 Zurich

www.zora.uzh.ch

Year: 2016

\title{
CME-Labor 46/Auflösung: Kardiale Parameter
}

Gawinecka, Joanna ; von Eckardstein, Arnold

DOI: https://doi.org/10.1024/1661-8157/a002395

Posted at the Zurich Open Repository and Archive, University of Zurich

ZORA URL: https://doi.org/10.5167/uzh-129306

Journal Article

Accepted Version

Originally published at:

Gawinecka, Joanna; von Eckardstein, Arnold (2016). CME-Labor 46/Auflösung: Kardiale Parameter. Praxis, 105(15):920-922.

DOI: https://doi.org/10.1024/1661-8157/a002395 


\section{PRAXIS}

\section{CME-Labor 46}

\section{Kardiale Parameter}

\section{Frage 1}

Welche Aussagen sind über BNP (B-Typ-natriuretisches Peptid) und NT-proBNP (aminoterminales Fragment des proBNP) richtig? (Mehrfachauswahl)

a) BNP/NT-proBNP eignen sich zur Diagnose oder Ausschluss einer Herzinsuffizienz.

b) BNP/NT-proBNP eignen sich zur Abschätzung der Prognose einer Herzinsuffizienz.

c) Die Referenzwerte sind alters- und geschlechtsabhängig.

d) Wegen der fehlenden Standardisierung der verschiedenen Assays sollen zur Bewertung die Hersteller-spezifischen Referenzwerte beachtet werden.

e) Körperliche Belastung hat keinen Einfluss auf BNP/NT-proBNP-Werte.

Richtige Antworten: a), b), c), d)

ProBNP wird als Folge von Wandstress der Ventrikel aus den Myozyten freigesetzt und in das bioaktive Fragment BNP und das inaktive N-terminale Bruchstück NT-proBNP gespalten. Ihre Konzentrationen im Blut steigen mit dem Ausmass und der Dauer der ventrikulären Dysfunktion des Herzens an. Beide Marker dienen zur Diagnose einer Herzinsuffizienz, zum Nachweis von milden Formen kardialer Funktionsstörungen und zur Beurteilung des Schwergrades einer Herzinsuffizienz nach der New York Heart Association (NYHA)-Klassifikation. Beide eignen sich auch gut für die Risikostratifizierung z.B. bei Patienten mit stabiler Angina pectoris oder Herzinfarkt.

Alter und Geschlecht sind die wichtigsten Faktoren, welche die BNP bzw. NT-proBNP-Konzentration beeinflussen. Frauen weisen höhere Plasmaspiegel auf als Männer, zudem steigen die Plasmaspiegel mit dem Alter an. Wegen der fehlenden Standarisierung der verschiedenen Assays beider Marker sollen zur Bewertung einer Herzinsuffizienz die Hersteller-spezifischen Angaben der Referenzwerte beachtet werden.

Körperliche Belastung kann auch bei Gesunden einen Anstieg von BNP bzw. NT-proBNP verursachen. Deswegen soll die Bestimmung beider Marker nicht nach körperlich belastenden Untersuchungen (z.B. Ergometrie) erfolgen.

\section{Frage 2}

Ein 60-jähriger Mann mit Diabetes mellitus und Raucheranamnese sucht seinen Hausarzt wegen akut aufgetretener Thoraxschmerzen und Atemnot auf. Die Symptomatik trat vor 4 Stunden auf. Das RuheEKG ist unauffällig und die Messung von kardialem Troponin mit einem point-of-care-(POC)-Gerät zeigt keine Troponin-Erhöhung.

Welche Aussage trifft zu? (Einfachauswahl) 
a) Ein Herzinfarkt kann ausgeschlossen werden, weil das EKG unauffällig sind.

b) Ein Herzinfarkt liegt sicher vor, weil die klinische Symptomatik eindeutig ist und kardiovaskuläre Risikofaktoren vorhanden sind.

c) Ein Herzinfarkt kann nicht ausgeschlossen werden, deshalb muss der Patient für weitere Abklärungen an ein Spital überwiesen werden.

d) Ein Herzinfarkt kann ausgeschlossen werden, weil kardiales Troponin nicht erhöht ist.

e) Ein Herzinfarkt kann nicht ausgeschlossen werden, weil die Symptomatik vor weniger als 6 Stunden begann. Der Patient sollte beim Hausarzt bleiben, sodass kardiales Troponin zwei Stunden später (6 Stunden nach Symptombeginn) nochmals gemessen werden kann.

\section{Richtige Antwort: c)}

Gemäss der aktuellen Definition ist die Voraussetzung für die Diagnose eines Herzinfarktes der Anstieg und/oder Abfall eines kardialen Troponins mit mindestens einem Wert über der 99. Perzentile eines Referenzkollektivs. Zusätzlich soll mindestens eines der folgenden Kriterien zutreffen: klinische Symptome der kardialen Ischämie, EKG-Veränderungen, bildgebender Nachweis des Verlustes von vitalem Myokard oder einer regionalen Bewegungsstörung der Herzwand oder Nachweis des intrakoronaren Thrombus durch Angiographie oder Autopsie ${ }^{1}$.

Wenn ausschliesslich die Kriterien der ST-Strecken-Hebung im EKG angewendet werden, ergibt sich für den Herzinfarkt eine Sensitivität von nur zirka 50\%. Wenn aber die ischämietypischen Formen der ST-T-Segments, ST-Strecken-Senkungen und Veränderungen des QRS-Komplexes mit einbezogen werden, steigt die Sensitivität des EKG auf zirka 90\%.

Für die Diagnostik des Herzinfarktes empfiehlt die Europäische Gesellschaft für Kardiologie das 3Stunden-Intervall für die Troponin-Bestimmung. Allerding gilt dieses Zeitintervall nur wenn hoch sensitive Troponin-Assays angewendet werden. Falls herkömmliche Assays angewendet werden, soll das längere 6-Stunden-Zeitintervall eingehalten werden. Ein Assay wird als hoch sensitiv eingestuft, wenn er eine analytische Impräzision von $<10 \%$ an der 99\%-Perzentile des gesunden Referenzkollektivs aufweist und wenn bei mehr als 50\% des gesunden Referenzkollektivs eine Troponin-Konzentration oberhalb der Nachweisgrenze bestimmt werden kann. Mit wenigen Ausnahmen werden die Troponin-Assays auf POC-Geräten nicht als hoch-sensitive Troponin-Assays klassifiziert.

Bei einer Symptomatik, die weniger als 6 Stunden andauert, kann ein Herzinfarkt sicher ausgeschlossen werden, wenn der hoch-sensitive Troponin-Wert bei der ersten Messung kleiner als das 99. Perzentil eines Referenzkollektivs ist und wenn die nach 3 Stunden wiederholte Messung einen unveränderten Wert liefert. Zudem muss der Patient wieder schmerzfrei und der berechnete GRACE-Risikoscore kleiner als 140 sein $^{1}$.

\section{$\underline{\text { Frage } 3}$}

Welche Erkrankungen können mit erhöhten BNP bzw. NT-proBNP-Werten einhergehen? (Mehrfachauswahl)

a) Pulmonale Hypertonie

b) Akuter Herzinfarkt

c) Cholangitis

d) Niereninsuffizienz

e) Leberzirrhose mit Aszites

Richtige Antwort: a), b), d), e) 
Bei der pulmonalen Hypertonie spiegeln die BNP bzw. NT-proBNP-Werte den Wandstress des rechten Ventrikels und die daraus folgende Beeinträchtigung der rechtsventrikulären Funktion wider.

Bei Patienten mit akutem Herzinfarkt kommt zu einem raschen Anstieg von BNP bzw. NT-proBNP und der Gipfelwert ist proportional zur Grösse des Infarktes. Die erhöhten Werte könnten bis 3 Monate nach dem Ereignis persistieren und reflektieren das Remodeling des Herzens.

Die wichtigste Ursache der Erhöhung von BNP bzw. NT-proBNP-Werten bei einer Niereninsuffizienz ist die Hypertrophie des linken Ventrikels. Zusätzlich können BNP bzw. NT-proBNP-Werte aufgrund einer reduzierten renalen Clearance erhöht sein.

Bei Patienten mit einer Leberzirrhose entwickelt sich bei Fortschreiten der Erkrankung eine hyperdynamische Zirkulation mit einem erniedrigtem Gefässwinderstand und erhöhtem Herzminutenvolumen (zirrhotische Kardiomyopathie). Die Erhöhung von BNP bzw. NT-proBNPWerten bei der Leberzirrhose korreliert gut mit dem Schweregrad der Erkrankung.

\section{Frage 4}

Welche Aussagen über die kardialen Troponine sind nicht richtig? (Mehrfachauswahl)

a) Eine Troponin-Konzentration grösser als die 99. Perzentile eines gesunden Referenzkollektivs beweist bei einem symptomatischen Patienten einen Herzinfarkt.

b) Es gibt verschiedene Troponin I Assays, deren Messergebnisse übereinstimmen.

c) Bei scheinbar gesunden Personen sind Troponin-Werte nicht messbar.

d) Eine Niereninsuffizienz beeinflusst die Troponin-Konzentration im Blut nicht.

e) Hoch sensitive Troponin-Assays haben einen Variationskoeffizient von >10\% bei der 99 . Perzentile eines gesunden Referenzkollektivs.

Richtige Antwort: b), e)

a) Da viele andere Konditionen mit Myokardschaden, z.B. hypertrophe Kardiomyopathie, Koronarspasmus, Myokarditis, hochgradige Lungenembolie, Sepsis oder kardiale Kontusionen, mit erhöhten Troponin-Werten einhergehen, reicht ein erhöhter Troponin-Wert nicht, um die Diagnose eines Herzinfarkts sicher zu stellen. Gemäss der Definition eines Herzinfarktes sollen die Troponin-Werten in seriellen Messungen eine ansteigende oder abfallende Dynamik zeigen.

b) Es gibt viele kommerzielle Troponin I Assays mit Antikörpern gegen diverse Epitope des Troponin I. Zudem zirkulieren verschiedene Troponin I Fragmente, welche durch die verschiedenen Immunoassays im unterschiedlichen Ausmass mitgemessen werden. Dies erschwert die Standardisierung von Troponin I Assays. Entsprechend weichen die Messergebnisse verschiedener Troponin I Assays bisweilen stark voneinander ab. Bei Troponin T besteht zwar grundsätzlich dasselbe Problem. Dagegen gibt es aus lizenzrechtlichen Gründen keine grosse Vielfalt von Troponin T Assays.

c) Bei Anwendung von hoch-sensitiven Assays können sogar bei gesunden Personen TroponinWerte gemessen werden. Es wurde allerdings gezeigt, dass auch Werte unterhalb den $99 \%$ Perzentile mit einer erhöhten 5-Jahresmortalität assoziiert sind ${ }^{2}$.

d) Bei Patienten mit einer Niereninsuffizienz werden vor allem erhöhte Werte von Troponin T gemessen. Die Ursache ist nicht vollständig geklärt, aber es wurde gezeigt, dass es sich um eine Akkumulation von Troponin T-Fragmenten durch eine verminderte renale Clearance handelt ${ }^{3}$. Nichtsdestoweniger hat sowohl die Troponin T- als auch Troponin I-Erhöhung bei niereninsuffizienten Patienten eine prognostische Bedeutung. Sie ist mit einer erhöhten Morbidität und Mortalität aufgrund von kardiovaskulären Ereignissen assoziiert.

e) Um die ansteigende oder abfallende Dynamik der Troponin-Werte in seriellen Messungen sicherzustellen, müssen die Troponin-Assays eine hohe Präzision aufweisen. Deswegen wurde gefordert, dass die Präzision der Messung bei der 99. Perzentile eines gesunden 
Referenzkollektivs ein Variationskoeffizient von $<10 \%$ besitzt. Troponin-Assays mit einem Variationskoeffizient $>20 \%$ bei der 99 . Perzentile sollen nicht für die Diagnostik eines Herzinfarktes verwendet werden.

\title{
Literatur
}

1. 2015 ESC Guidelines for the management of acute coronary syndromes in patients presenting without persistent ST-segment elevation http://www.escardio.org/Guidelines-\&Education/Clinical-Practice-Guidelines/Acute-Coronary-Syndromes-ACS-in-patientspresenting-without-persistent-ST-segm)

2. Eggers KM, Jaffe AS, Lind L, Venge P, Lindahl B. Value of cardiac troponin I cutoff concentrations below the 99th percentile for clinical decision-making. Clin Chem. 2009 Jan;55(1):85-92.

3. Diris JH, Hackeng CM, Kooman JP, Pinto YM, Hermens WT, van Dieijen-Visser MP. Impaired renal clearance explains elevated troponin $\mathrm{T}$ fragments in hemodialysis patients. Circulation. 2004 Jan 6;109(1):23-5

\author{
Korrespondenzadresse \\ Dr. Joanna Gawinecka \\ Institut für Klinische Chemie \\ UniversitätsSpital Zürich \\ Rämistrasse 100 \\ 8091 Zürich \\ joanna.gawinecka@usz.ch
}

